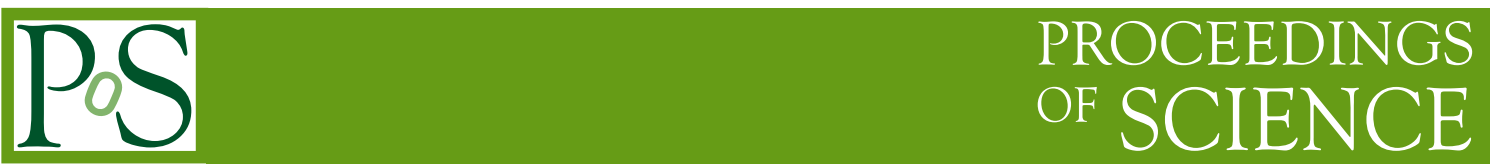

\title{
Pion and photon production in heavy ion collisions
}

\author{
Gabor David*† \\ $B N L, U S A$ \\ E-mail: david@bnl.gov
}

\begin{abstract}
Measurement of neutral pions and direct photons are closely connected experimentally, on the other hand they probe quite different aspects of relativistic heavy ion collisions. In this short review of the $\pi^{0}$ results from the PHENIX experiment at RHIC our focus is on the $\phi$-integrated nuclear modification factor, its energy and system size dependence, and the impact of these results on parton energy loss models. We also discuss the current status of high $p_{T}$ and thermal direct photon measurements both in $p+p$ and $\mathrm{Au}+\mathrm{Au}$ collisions. Recognizing the advantages of measuring not only the "signal", but also all the "references" needed for proper interpretation in the same experiments (with same or similar systematics) we argue that RHIC should regularly include $d+\mathrm{A}$ and even $d+d$ collisions into its system size and energy scan.
\end{abstract}

High-pT Physics at LHC - Tokaj'08

March 16 - 192008

Tokaj, Hungary

\footnotetext{
*Speaker.

${ }^{\dagger}$ This work has been supported by the Office of Science of the United States Department of Energy
} 


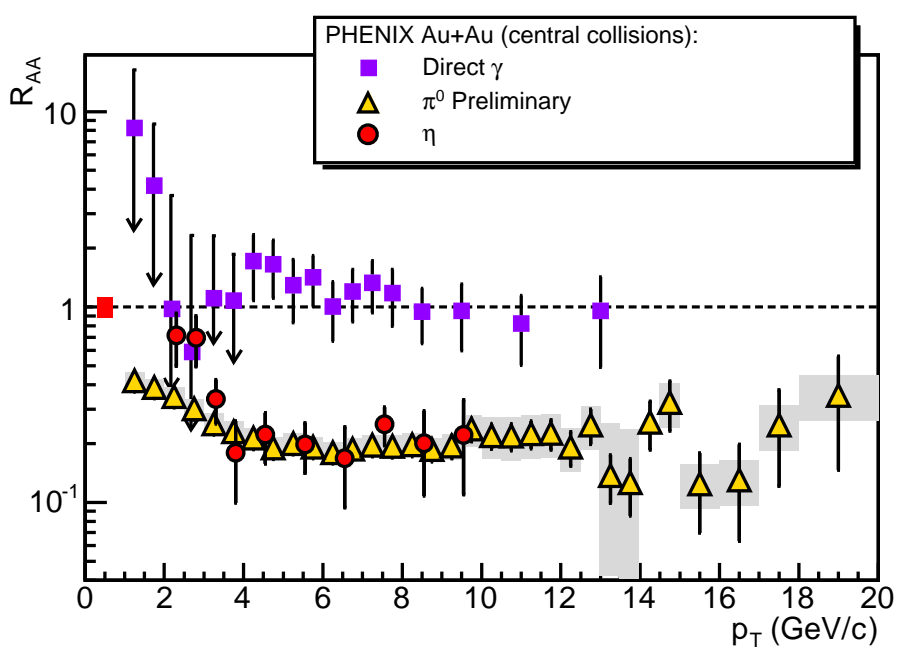

Figure 1: "Historic" nuclear modification factors $R_{A A}$ vs $p_{T}$ in 200GeV/A Au+Au collisions. Squares: direct photons (no apparent suppression [丹], published 2005). Triangles and circles: preliminary results on $\pi^{0}$ and $\eta$, also from 2005.

\section{Introduction}

One of the predicted signals of the formation of a quark-gluon plasma (QGP) in relativistic heavy ion collisions was the in-medium energy loss of hard scattered partons, leading to "jet quenching" and manifesting itself in a drastic reduction of high $p_{T}$ hadron production in relativistic heavy ion collisions, as compared to the production rate in $p+p$ scaled by the nuclear thickness function $T_{A A}$ to account for the increased probability of hard scattering. The effect has been characterized by the nuclear modification factor $R_{A A}$ defined as

$$
R_{A A}^{\alpha}\left(p_{T}\right)=\frac{1}{N_{e v t}} \frac{d^{2} N_{A A}^{\alpha} / d p_{T} d y}{\left\langle T_{A A}\right\rangle d^{2} \sigma_{p p}^{\alpha} / d p_{T} d y}
$$

for an arbitrary particle type $\alpha$. In the absence of nuclear effects $R_{A A}=1$, otherwise it is enhanced ( $R_{A A}>1$, like in the "Cronin-effect") or suppressed $\left(R_{A A}<1\right.$ like in jet quenching.) The first major discovery at RHIC was that in central $\mathrm{Au}+\mathrm{Au}$ collisions hadrons were strongly suppressed [1] (see Fig. 1), in accordance with the predicted energy loss of partons in the hot, dense medium. Lack of suppression in $\mathrm{d}+\mathrm{Au}$ collisions [3] (where we assume no such medium is formed) supported the conclusion that the jet suppression was indeed a final state effect, as opposed to some drastic changes in the initial state (parton distribution functions). The first observation that direct photons were not suppressed, not even in the most central Au+Au collisions [ [ this first, qualitative picture coherent, by showing that the crucial step in forming $R_{A A}$ - scaling $p+p$ cross sections by $\left\langle T_{A A}\right\rangle$ to obtain the expected Au+Au yields - makes sense: photons will not interact with the medium $\left(\alpha_{e m}<<\alpha_{s}\right)$, so if the $\left\langle T_{A A}\right\rangle$ scaling is the right concept, the direct photon $R_{A A}$ should be unity - and indeed, it is (see Fig 1).

While very useful and providing many early insights, causing a flurry of theoretical activities rarely seen before, the above picture turned out to be only rudimentary. As RHIC experiments moved "from the discovery phase to the exploration phase" more precise data became available, 
with higher statistics, extended $p_{T}$ ranges and more complex physics quantities (like two- and three-particle correlations, azimuthal anisotropies, etc. Equally important, RHIC launched a systematic energy and colliding species scan. This made measuring excitation functions in the same collider and within the same experiments possible. The collider environment ensures that multiplicities at midrapidity rise only slowly with $\sqrt{s_{N N}}$, and measuring in the same experiment means that most systematic errors will be similar - even if individual measurements are not very precise, the evolution with energy and/or system size can be established quite accurately. Increased accuracy of the data made comparisons with theories more meaningful, put some constraints on free parameters [5] and gave the first experimental indications at what region of the phase diagram the transition to the strongly coupled quark gluon plasma might occur. Almost needless to say that in the process our initial, relatively simple picture became richer (much more complicated).

\section{Pion suppression systematics}

So far four aspects of the $\phi$-integrated $R_{A A}$ systematics have been studied at RHIC. First, the magnitude and shape of $R_{A A}\left(p_{T}\right)$ at the highest available transverse momenta - namely, whether it remains constant, consistent with a constant fractional energy loss picture, or rises with increasing $p_{T}$, as predicted by most parton energy loss models [5]. These studies gave the first quantitative constraints on free parameters of models, like initial gluon density or the transport coefficient of the PQM [6] model $\left\langle\hat{q}=\mu^{2} / \lambda\right\rangle=13.2_{-3.2}^{+2.1} \mathrm{GeV}^{2} / \mathrm{fm}$, where $\mu$ is the average transverse momentum transfer to the medium per mean free path $\lambda$. PQM - which includes radiative loss, a static medium, no initial state multiple scattering and unmodified PDFs - predicts a slow rise of $R_{A A}$ with $p_{T}$, as do other models with very different assumptions [5]. In light of this it is interesting, that the data are best described by a constant fit which in turn for the power law spectra of high $p_{T}$ pions would imply a constant fractional energy loss (constant $S_{\text {loss }}=\Delta p_{T} / p_{T}$ fractional shift of the spectrum). These statements are based on data taken in RHIC Run-4 (2004), which still have large errors on the high $p_{T}$ points, results from the much higher statistics 2007 data should be available soon.

Second, the evolution of $R_{A A}$ with collision centrality has been studied. One very interesting and non-trivial observation was that the shape of the high $p_{T} \pi^{0}$ spectra is virtually unchanged from $p+p$ to the most central Au+Au collisions. PHENIX found [7] that the exponent of the power law function fitting the high $p_{T}$ part of the spectra $\left(\propto p_{T}^{n}\right)$ varies from $n=-8.22 \pm 0.09$ in $p+p$ to $n=-8.00 \pm 0.012$ in the most central (0-5\%) Au+Au collisions. This combined with the fact that $R_{A A}$ in the most central collisions is essentially flat (constant) means that $R_{A A}$ is constant at all centralities to the best of our current knowledge which in turn means that $R_{A A}$ integrated above a certain $p_{T}$ is a useful quantity to characterize the onset and evolution of jet quenching. This is shown on Fig. 2. Fitting the points with a function motivated by the constant fractional energy loss (i.e the energy loss increases with $\left.p_{T}\right) R_{A A}=\left(1-S_{0} N_{\text {part }}^{a}\right)^{n-2}$ the exponent $a$ is found to be $a=0.58 \pm 0.07$ for $p_{T}>5 \mathrm{GeV} / \mathrm{c}$ and $a=0.56 \pm 0.10$ for $p_{T}>10 \mathrm{GeV} / \mathrm{c}$ which is in reasonable (albeit not perfect) agreement with the $a \approx 2 / 3$ predictions of the PQM [6] and GLV [8] models. Note that in the "constant fractional energy loss" picture $R_{A A}$ depends on the slope (exponent $n$ ) of the power-law $p_{T}$ spectrum which in turn depends on the per-nucleon collision energy $\sqrt{s_{N N}}$.

Therefore, the third issue is what can be learned from the energy dependence of $R_{A A}$, and the first answer is given on Fig. 3 which shows the nuclear modification factors measured at RHIC in 


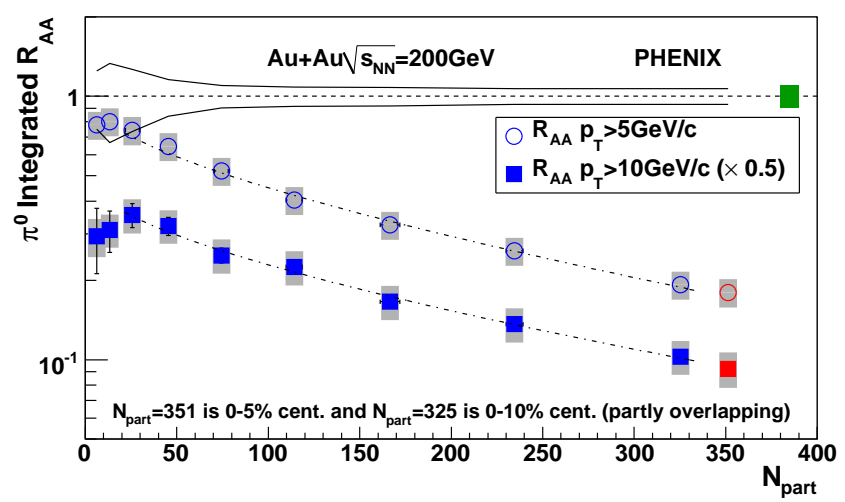

Figure 2: Integrated $R_{A A}$ for $\pi^{0}$ as a function of collision centrality expressed in terms of $N_{\text {part }}$. The last two points correspond to overlapping centrality bins $0-10 \%$ and $0-5 \%$. See text for an explanation of the fits.

the most central $\mathrm{Cu}+\mathrm{Cu}$ collisions at $\sqrt{s_{N N}}=22.4,62.4$ and 200GeV. Instead of suppression the lowest energy data show a substantial enhancement of $R_{A A}$ in line with the well-known "Cronin-effect"

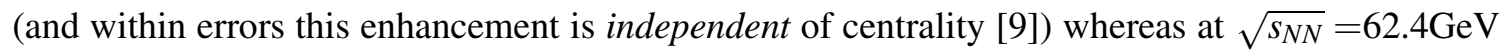
the pions are already significantly suppressed. Of course it does not follow that the onset of jet quenching - presumably due to QGP formation - happens necessarily between $\sqrt{s_{N N}}=22.4$ and $62.4 \mathrm{GeV}$; all we can say is that the quenching mechanism overtakes the Cronin-effect in this region. Being a consequence of the initial $k_{T}$ smearing of partons the Cronin-effect is present already in cold nuclei and could be unfolded from the $\sqrt{s_{N N}}$-dependence of $R_{A A}$ if $p+\mathrm{A}(d+\mathrm{A})$ data were available from RHIC in the entire available collision energy range. In the author's opinion taking $d+\mathrm{A}$ data (along with $\mathrm{A}+\mathrm{A}$ and $p+p$ for reference) should be part of the planned "energy scan" at RHIC. - Since pions are strongly suppressed in $\mathrm{Cu}+\mathrm{Cu}$ already at $\sqrt{s_{N N}}=62.4 \mathrm{GeV}$, it is not surprising that they are also suppressed in $\mathrm{Au}+\mathrm{Au}$, in fact, almost as strongly as at 130 or $200 \mathrm{GeV}$.

Finally, the fourth issue is how does $R_{A A}$ depend on the (size of the) colliding systems at any given energy. A comparison of $\sqrt{s_{N N}}=200 \mathrm{GeV}$ central $\mathrm{Cu}+\mathrm{Cu}$ collisions to mid-central $\mathrm{Au}+\mathrm{Au}$ (such that the number of participating nucleons $N_{\text {part }}$ is the same in both cases albeit the geometry is not) shows similar values of $R_{A A}$. It would be interesting to know which is the lightest, symmetric heavy ion system where jet quenching at $\sqrt{s_{N N}}=200 \mathrm{GeV}$ would disappear.

So far we discussed only the $\phi$-integrated $R_{A A}$. For any given dataset neglecting azimuthal dependence results in higher precision of the measurement itself (better statistics and no systematic errors related to the determination of the reaction plane) but it also hides any information encoded in azimuthal anisotropies of $R_{A A}$ due to the overlap geometry of the colliding nuclei. Such anisotropies clearly exist: after all, some of the most spectacular observations at RHIC are the large "elliptic flow" of hadrons and the quark number scaling thereof. Indeed, when studying $R_{A A}(\Delta \Phi)$ where $\Delta \Phi$ is the azimuthal angle with respect to the reaction plane, determined event-by-event, we observe a rich structure [10]: jet quenching varies substantially with the average pathlength of the parton in the medium. Back-to-back dihadron (or more ambitiously, dijet) correlations, and particularly the "golden channel", photon-hadron (photon-jet) correlations have also been advocated and now measured - as tools of "jet tomography". It has been argued [11] that single hadron spectra at high $p_{T}$ are dominated by surface emission, therefore, $R_{A A}$ is barely sensitive to the medium 


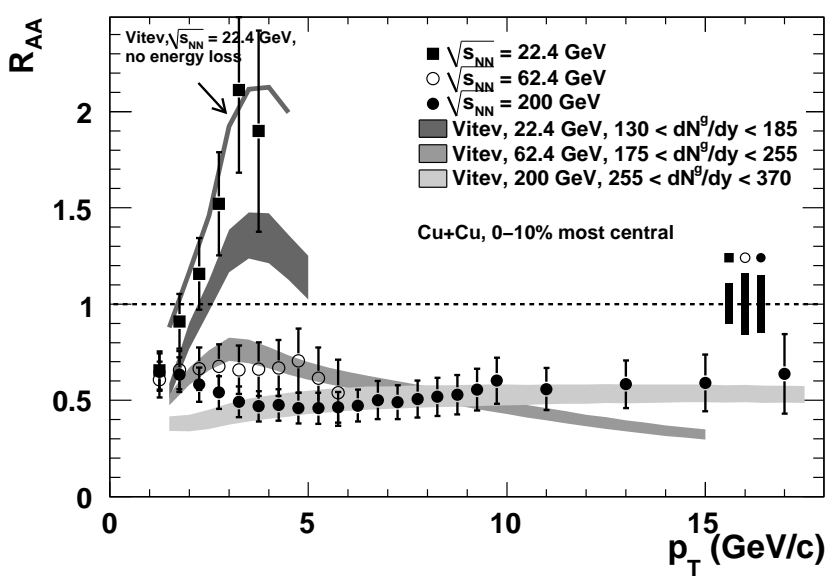

Figure 3: Nuclear modification factors for the most central $\mathrm{Cu}+\mathrm{Cu}$ collisions [9] for $\sqrt{s_{N N}}=22.4,62.4$ and $200 \mathrm{GeV}$ compared to theoretical calculations [12].

deep inside the collision volume. On the other hand back-to-back correlations force one of the partons to go through the medium (with the exception of "tangential" emissions) thus the dihadron suppression $I_{A A}$ is more sensitive to the input parameters of theories like initial energy or gluon density. This is certainly true as long as the uncertainties of both theory and experimental data are negligible: a multidifferential quantity always provides more constraints. However, theories usually have uncertainties (even if often unstated, regrettably), and experimental data always have them and it is hard to imagine a situation in which using a given dataset a multidifferential quantity could be measured more precisely than a single differential. Just the opposite. The quantitative question then is: does the increased sensitivity on the theory side compensate for the inevitable loss of precision in the data? The answer varies from case to case.

\section{Direct photons}

Direct photons - defined as those not originating from final state hadron decays - offer unique possibilities to study heavy ion collisions. First, there are physics mechanisms that produce direct photons at each stage of the collision, thus they have the potential to provide information on the entire evolution, "history" of the collision. Equally important, once produced, they leave the interaction region mostly unaltered $\left(\alpha_{e m}<<\alpha_{s}\right)$, so the information they carry is unbiased. Unfortunately, the very same properties make them challenging to measure (photons from hadron decays provide a large background, particularly at low $p_{T}$ ) and their "message" is hard to decypher because it is difficult (if not impossible) to deconvolute the contributions of individual mechanisms to the observed direct photon spectrum.

On Fig. 1 1 we already have seen the first measurement of direct photon $R_{A A}$ in central $\mathrm{Au}+\mathrm{Au}$ collisions at $\sqrt{s_{N N}}=200 \mathrm{GeV}$, showing that in the region where photons from primordial hard scattering were expected to dominate $\left(p_{T}>5-6 \mathrm{GeV} / c\right) R_{A A}$ is indeed consistent with unity. This was consistent with the picture that both the PDFs and the production cross section are unchanged, the only difference between $p+p$ and $\mathrm{Au}+\mathrm{Au}$ as far as hard scattering is concerned is the increased probability of an otherwise rare process; this increased probability is properly described by a 


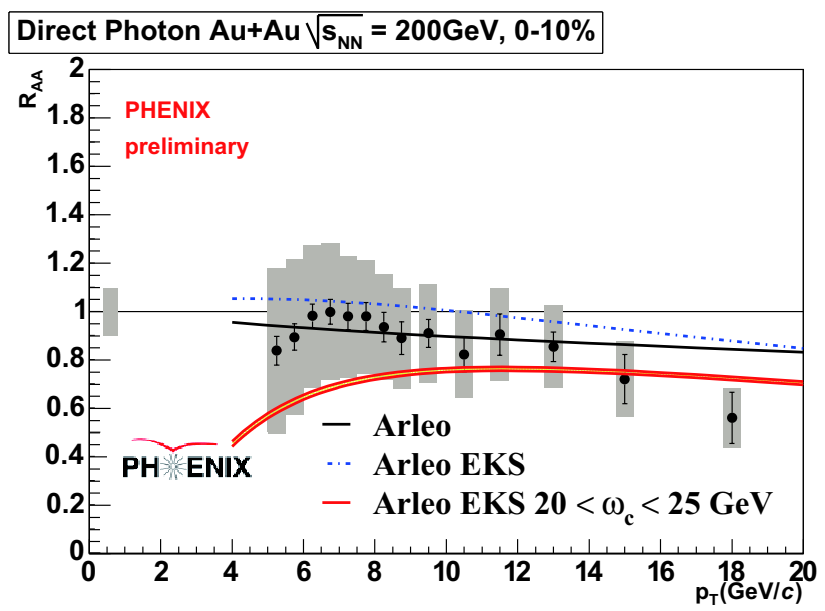

Figure 4: Direct photon $R_{A A}$ in central $\mathrm{Au}+\mathrm{Au}$ collisions. The denominator is a fit to the PHENIX preliminary Run-5 $p+p$ data. Theoretical curves are LO calculations from [15]. The solid line shows the pure isospin effect, the dash-dotted line is the combined effect of isospin and (anti)shadowing (EKS), finally the band at the botoom combines isospin and antishadowing with photon quenching.

straightforward geometric overlap integral (the nuclear thickness function $T_{A A}$ ), and the photons produced escape the collision region unaltered even if a dense, strongly interacting medium is formed which quenches parton jets. Although we have higher quality data now (in an extended range) and our understanding of the processes generating photons is much more nuanced, we should emphasize that at a very basic level, in first approximation, qualitatively the above picture is still correct.

What are the currently known/assumed sources of direct photons in $p+p$ and $\mathrm{Au}+\mathrm{Au}$ collisions? At leading order two processes generate photons, the quark-quark annihilation $(q \bar{q} \rightarrow g \gamma)$, which is suppressed at high $p_{T}$ due to the lack of valence antiquarks, and the dominant quark-gluon Compton-scattering $(q g \rightarrow q \gamma)$. Note that in the latter the photon balances the momentum of the original quark (modulo the initial $k_{T}$ smearing of the partons) and it comes out freely from the medium, whereas the quark will lose a significant fraction of its energy. Therefore, in back-to-back photon-jet correlations the photon "calibrates" the energy scale of the jet, which is important in "jet tomography" as well as in studying possible modifications of the fragmentation function in the presence of a medium. The probe itself is very clean and well understood - unfortunately the rates are quite low, due to the $\alpha_{e m}$ coupling.

At higher order fragmentation (or Bremsstrahlung) photons contribute both in $p+p$ and heavy ion collisions. In principle Compton and fragmentation photons are distinguishable (at least in $p+p$ ) because fragmentation photons should have enhanced, jet-like activity in a small cone around them (tracks or energy deposit that exceeds the level justified by the underlying event), whereas LO Compton photons should have none in their neighborhood: they are "isolated". There are predictions that at high $p_{T}$ fragmentation photons will be a larger fraction of all direct photons in $\mathrm{A}+\mathrm{A}$ collisions than in $p+p$. This is another reason why is it so important to understand $p+p$ processes as well as possible: they will serve as reference when we try to find new phenomena (new sources of direct photons) in A+A. First direct photon cross-sections in $\sqrt{s}=200 \mathrm{GeV} p+p$ collisions at RHIC 


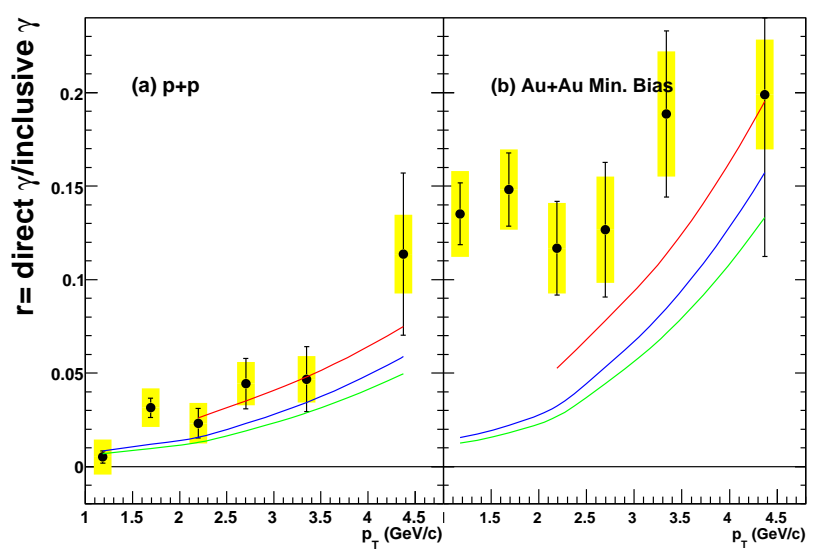

Figure 5: The fraction of the direct photon component as a function of $p_{T}$ in (a) $p+p$ and (b) $\mathrm{Au}+\mathrm{Au}(\mathrm{min}$. bias). The error bars and the error band represent the statistical and systematic uncertainties, respectively. The curves are from a NLO pQCD calculation.

were published in [13] followed by [14] with extended $p_{T}$ range, small systematic errors and detailed comparison to NLO pQCD calculations. Above $p_{T}>5 \mathrm{GeV} / c$ where the systematic errors of theory and experiment are comparable, the data are well described by the theory, including the fraction of isolated photons

Is $p+p$ the right reference at all? For pions it certainly is due to the isospin symmetry of the protons and neutrons of the nucleus. The same is not true for direct photons: due to the $\alpha_{e m}$ coupling the photon cross section is proportional to the sum of the squared quark charges $\Sigma e_{q}^{2}$ which is different for $p$ and $n$. This "isospin effect" causes a trivial shift of the direct photon $R_{A A}$ [15], depending on the nucleus and to some small extent on centrality; in the absence of any other medium or nuclear effect $R_{A A}$ should go about 10-15\% below unity in Au+Au collisions (see the solid curve on Fig. 4 , which shows the latest, still preliminary PHENIX results for photon $R_{A A}$ in the most central Au+Au collisions). Note that this trivial effect may either mask or enhance other mechanisms affecting $R_{A A}$. The clean way to generate direct photon $R_{A A}$ would be to compare to a proper mixture of photon cross sections in $p+p, p+n$ and $n+n$ collisions, which could be readily available at RHIC from $d+d$ collisions, since these three types of collisions could easily be tagged.

A recent overview of the possible (additional) photon production mechanisms in heavy ion collisions is given in [16]. At medium $p_{T}$ (corresponding to the $0.1<x<0.2$ region) anti-shadowing should enhance the photon yield. The large parton energy loss (deduced from $\pi^{0}$ suppression) means that fragmentation photons could be suppressed in the entire known $p_{T}$ range, unless the fragmentation functions themselves change in the presence of a medium (which is hard, but not impossible to measure in $\mathrm{Au}+\mathrm{Au}$ ). On the other hand the jet-photon conversion mechanism may increase high $p_{T}$ photon production. In this process a hard scattered quark interacts with a gluon or antiquark of the medium and the outcoming photon carries essentially the full momentum of the original parton (hence the name). Disentangling the individual contributions of these processes will not be easy, although we should point out that some mechanisms produce isolated, others give non-isolated photons, and their azimuthal asymmetries are also different (for instance jet-photon 
conversion should produce isolated photons with a negative "elliptic flow" $v_{2}<0$ which is quite unique since all other particles tested so far at RHIC exhibited $v_{2}>0$ ).

Low $p_{T}$ real photons (thermal region) are very hard to measure due to the overwhelming hadron decay background, however, "quasi-real" photons are accessible as low mass $e^{+} e^{-}$pairs. Using this technique PHENIX measured the fraction of direct photons in the total inclusive photon spectrum [17] (see Fig. 5) and found that while in $p+p$ the results are consistent with NLO pQCD calculations (there are no unexpected sources), in $\mathrm{Au}+\mathrm{Au}$ there is a statistically significant $10 \%$ excess at low $p_{T}$.

In summary, after the early, exciting discoveries RHIC experiments entered the era of exploration. Data became more precise enabling detailed comparisons to theories and setting meaningful constraints on their free parameters. Taking advantage of the flexibility of the accelerator a deliberate study of energy- and system-size dependence of the new phenomena is underway. The lively cooperation of theorists and experimentalists (strengthened by some new initiatives) will certainly lead to our much deeper understanding of the strongly coupled quark-gluon plasma.

\section{References}

[1] K. Adcox et al., Phys. Rev. Lett. 88 (2002) 022301

[2] S. S. Adler et al., Phys. Rev. Lett. 91 (2003) 072301

[3] S. S. Adler et al., Phys. Rev. Lett. 91 (2003) 072303

[4] S. S. Adler et al., Phys. Rev. Lett. 94 (2005) 232301

[5] A. Adare et al., Phys. Rev. C 77 (2008) 064907

[6] A. Dainese, C. Loizides, G. Paic, Eur. Phys. J. C38 (2005) 461; C. Loizides, Eur. Phys. J. C49 (2007) 339

[7] A. Adare et al., arXiv:0801.4020, to be published in Phys. Rev. Lett.

[8] M. Gyulassi, P. Levai, I. Vitev, Phys. Rev. Lett. 85 (2000) 5535

[9] A. Adare et al., Phys. Rev. Lett. 101 (2008) 162301

[10] S. S. Adler et al., Phys. Rev. C76 (2007) 034904

[11] H. Zhang, J. F. Owens, E. Wang, X.-N. Wang, Phys. Rev. Lett. 98 (2007) 212301

[12] I. Vitev Phys. Lett. B639 (2006) 38

[13] S.S. Adler et al., Phys. Rev. D 71 071102(R) (2005)

[14] S.S. Adler et al., Phys. Rev. Lett. 98, (2007) 012002

[15] F. Arleo, JHEP09 (2006) 015

[16] S. Turbide, C. Gale, E. Frodermann, U. Heinz, Phys. Rev. C 77, (2008) 024909

[17] A. Adare et al., arXiv:0804.4168 